MATHEMATICS OF COMPUTATION

Volume 67, Number 221, January 1998, Pages 323-336

S $0025-5718(98) 00882-5$

\title{
QUADRATURE FORMULAE USING ZEROS OF BESSEL FUNCTIONS AS NODES
}

\author{
RIADH BEN GHANEM
}

\begin{abstract}
A gaussian type quadrature formula, where the nodes are the zeros of Bessel functions of the first kind of order $\alpha(\Re(\alpha)>-1)$, was recently proved for entire functions of exponential type. Here we relax the restriction on $\alpha$ as well as on the function. Some applications are also given.
\end{abstract}

\section{IntRodUCtion AND STATEMENT OF THE RESULTS}

It was proved by Boas [4] that if $f$ is an entire function of exponential type $2 \tau>0$ belonging to $L^{1}(-\infty, \infty)$, then

$$
\int_{-\infty}^{\infty} f(x) d x=\frac{\pi}{\tau} \sum_{k=-\infty}^{\infty} f\left(\frac{(2 k-1) \pi}{2 \tau}\right) .
$$

This formula is equivalent to the following:

$$
\int_{0}^{\infty}(f(x)+f(-x)) d x=\frac{\pi}{\tau} \sum_{k=1}^{\infty}\left(f\left(\frac{(2 k-1) \pi}{2 \tau}\right)+f\left(-\frac{(2 k-1) \pi}{2 \tau}\right)\right),
$$

since we may make the decomposition $f(x)=(f(x)+f(-x)) / 2+(f(x)-f(-x)) / 2$.

In this form, it is clear by applying (1) to $f(z)+f(-z)$, that it is enough to suppose that $f(z)+f(-z)$ is entire of exponential type $2 \tau$ such that $f(x)+f(-x)$ belongs to $L^{1}(0, \infty)$ to obtain (1) without imposing any condition on $f(z)-f(-z)$.

Let $J_{\alpha}(z)$ be the Bessel function of the first kind of order $\alpha$. It may be recalled that the function

$$
G_{\alpha}(z):=\frac{J_{\alpha}(z)}{z^{\alpha}}=\sum_{k=0}^{\infty}(-1)^{k} \frac{z^{2 k}}{2^{\alpha+2 k} k ! \Gamma(k+\alpha+1)}
$$

is an even entire function of exponential type 1 . We denote by $j_{k}=j_{k}(\alpha)$, $k= \pm 1, \pm 2, \ldots$, the nonnull zeros of $G_{\alpha}(z)$ which are all simple, ordered such that $j_{-k}=-j_{k}$ and $0<\left|j_{1}\right| \leq\left|j_{2}\right| \leq \ldots$. Since $G_{\alpha}(0)=\frac{1}{2^{\alpha} \Gamma(\alpha+1)}$, the only values of $\alpha$ for which $G_{\alpha}(0)$ is equal to zero are $-1,-2, \ldots$.

Formula (1) has recently been extented using the zeros of $G_{\alpha}(z)$ as nodes [5].

Received by the editor March 27, 1996 and, in revised form, September 11, 1996.

1991 Mathematics Subject Classification. Primary 65D32, 41A55, 33C10.

Key words and phrases. Quadrature formulae, entire functions, Bessel functions.

(C) 1998 American Mathematical Society 
Theorem A. Let $\Re(\alpha)>-1$. For all functions $f$ of exponential type $2 \tau$ such that $f(x)=O\left(|x|^{-\delta}\right), x \rightarrow \pm \infty$, with $\delta>2 \Re(\alpha)+2$, we have

$$
\int_{0}^{\infty} x^{2 \alpha+1}(f(x)+f(-x)) d x=\frac{2}{\tau^{2 \alpha+2}} \sum_{k=1}^{\infty} \frac{j_{k}^{2 \alpha}}{\left(J_{\alpha}^{\prime}\left(j_{k}\right)\right)^{2}}\left(f\left(\frac{j_{k}}{\tau}\right)+f\left(-\frac{j_{k}}{\tau}\right)\right) .
$$

This result is in fact valid under weaker integrability conditions [8].

Theorem B. If $\alpha>-1$, then (2) holds for every entire function $f$ of exponential type $2 \tau$ such that $x^{2 \alpha+1}(f(x)+f(-x))$ belongs to $L^{1}[0, \infty)$. Besides, the series on the right-hand side of (2) is absolutely convergent.

If $f$ is integrable on $[1, X]$ for all $X>1$ and $\lim _{X \rightarrow \infty} \int_{1}^{X} f(x) d x$ exists, then we denote the limit by $\int_{1}^{\rightarrow \infty} f(x) d x$ and say that $f$ is integrable in the sense of Cauchy on $[1, \infty)$. If $f$ is integrable on $[\delta, 1]$ for all $\delta \in(0,1)$ and $\lim _{\delta \rightarrow 0} \int_{\delta}^{1} f(x) d x$ exists, then we denote the limit by $\int_{\rightarrow 0}^{1} f(x) d x$ and say that $f$ is integrable in the sense of Cauchy on $(0,1]$. If $f$ is integrable in the sense of Cauchy on $[1, \infty)$ and on $(0,1]$, then we say that $f$ is integrable in the sense of Cauchy on $(0, \infty)$ and denote the integral by

$$
\int_{\rightarrow 0}^{\rightarrow \infty} f(x) d x
$$

We remark that if $f$ belongs to $L^{1}(0, \infty)$, then

$$
\int_{\rightarrow 0}^{\rightarrow \infty} f(x) d x=\int_{0}^{\infty} f(x) d x .
$$

Changing convergence in $L^{1}[0, \infty)$ by convergence in the sense of Cauchy on $(0, \infty)$, the following result was proved in $[8]$.

Theorem C. Let $\alpha>-1$ and $f$ be an entire function of exponential type $\sigma<2 \tau$ such that $x^{2 \alpha+1}(f(x)+f(-x))$ is integrable in the sense of Cauchy on $(0, \infty)$. Then

$$
\int_{\rightarrow 0}^{\rightarrow \infty} x^{2 \alpha+1}(f(x)+f(-x)) d x=\frac{2}{\tau^{2 \alpha+2}} \sum_{k=1}^{\infty} \frac{j_{k}^{2 \alpha}}{\left(J_{\alpha}^{\prime}\left(j_{k}\right)\right)^{2}}\left(f\left(\frac{j_{k}}{\tau}\right)+f\left(-\frac{j_{k}}{\tau}\right)\right)
$$

if the series on the right is convergent.

Since in Theorem A it is supposed that $\Re(\alpha)>-1$, the question arises if this theorem would hold for any complex number $\alpha$, and if Theorems B and C would hold under the same condition on $\alpha$. The answer is affirmative when $\alpha$ is not a negative integer, as we will see later.

The particular case $\alpha=\frac{-1}{2}$ of Theorem B, was proved in [6, Theorem 1] where formula (3) was in this case replaced by an equivalent formula which we may obtain by a translation, and later it was shown in [9, Corollary 4] that in this theorem the condition regarding the convergence of the series is superfluous. So it appears natural to expect Theorem $\mathrm{C}$ to hold without this condition.

We are now ready to state our main results. 
Theorem 1. Let $\alpha \in \mathbb{C} \backslash\{-1,-2, \ldots\}$ and $p$ a nonnegative integer. If $f$ is a function such that $f(z)+f(-z)$ is entire of exponential type $\sigma<2 \tau$ and $x^{2 \Re(\alpha)+2 p+1}(f(x)+f(-x))$ is integrable in the sense of Cauchy on $(0, \infty)$, then

$$
\begin{aligned}
\int_{\rightarrow 0}^{\rightarrow \infty} & x^{2 \alpha+2 p+1}(f(x)+f(-x)) d x \\
= & \frac{2}{\tau^{2 \alpha+2 p+2}} \sum_{k=1}^{\infty} \frac{j_{k}^{2 p}}{\left(G_{\alpha}^{\prime}\left(j_{k}\right)\right)^{2}}\left(f\left(\frac{j_{k}}{\tau}\right)+f\left(-\frac{j_{k}}{\tau}\right)\right) .
\end{aligned}
$$

Theorem 2. Let $\alpha \in \mathbb{C} \backslash\{-1,-2, \ldots\}$ and $p$ a nonnegative integer. If $f$ is a function such that $f(z)+f(-z)$ is entire of exponential type $2 \tau$ and $x^{2 \alpha+2 p+1}(f(x)+$ $f(-x)$ ) belongs to $L^{1}[0, \infty)$, then

$$
\begin{aligned}
\int_{0}^{\infty} & x^{2 \alpha+2 p+1}(f(x)+f(-x)) d x \\
& =\frac{2}{\tau^{2 \alpha+2 p+2}} \sum_{k=1}^{\infty} \frac{j_{k}^{2 p}}{\left(G_{\alpha}^{\prime}\left(j_{k}\right)\right)^{2}}\left(f\left(\frac{j_{k}}{\tau}\right)+f\left(-\frac{j_{k}}{\tau}\right)\right) .
\end{aligned}
$$

Besides, the series on the right-hand side of (5) is absolutely convergent.

The particular case $p=0$ in (4) and (5) leads us to formula (3) and formula (2) respectively, since we have

$$
\frac{J_{\alpha}^{\prime}\left(j_{k}\right)}{j_{k}^{\alpha}}=G_{\alpha}^{\prime}\left(j_{k}\right) \text { for } k=1,2, \ldots
$$

The following result was also proved in [8].

Theorem D. If $f$ is an entire function of exponential type $\tau$ such that $|x|^{\alpha+\frac{1}{2}} f(x)$ $\in L^{2}(\mathbb{R})$ for some $\alpha>-1$, then

$$
\int_{-\infty}^{\infty}|x|^{2 \alpha+1}|f(x)|^{2} d x=\frac{2}{\tau^{2 \alpha+2}} \sum_{\substack{k=-\infty \\ k \neq 0}}^{\infty} \frac{\left|j_{k}\right|^{2 \alpha}}{\left(J_{\alpha}^{\prime}\left(\left|j_{k}\right|\right)\right)^{2}}\left|f\left(\frac{j_{k}}{\tau}\right)\right|^{2} .
$$

We remark using (6) that (7) is equivalent to

$$
\int_{-\infty}^{\infty}|x|^{2 \alpha+1}|f(x)|^{2} d x=\frac{2}{\tau^{2 \alpha+2}} \sum_{\substack{k=-\infty \\ k \neq 0}}^{\infty} \frac{1}{\left(G_{\alpha}^{\prime}\left(j_{k}\right)\right)^{2}}\left|f\left(\frac{j_{k}}{\tau}\right)\right|^{2},
$$

since the zeros of $G_{\alpha}(z)$ are all real for $\alpha>-1$ and $G_{\alpha}^{\prime}\left(-j_{k}\right)=-G_{\alpha}^{\prime}\left(j_{k}\right)$. So, in view of Theorem 2, we may extend Theorem $\mathrm{D}$ as follows:

Theorem 3. Let $\alpha \in \mathbb{R} \backslash\{-1,-2, \ldots\}$ and let $p$ be a nonnegative integer. If $f$ is an entire function of exponential type $\tau$ such that $x^{\alpha+p+\frac{1}{2}} f(x) \in L^{2}(\mathbb{R})$, then we have

$$
\int_{-\infty}^{\infty}|x|^{2 \alpha+2 p+1}|f(x)|^{2} d x=\frac{2}{\tau^{2 \alpha+2 p+2}} \sum_{\substack{k=-\infty \\ k \neq 0}}^{\infty} \frac{j_{k}^{2 p}}{\left(G_{\alpha}^{\prime}\left(j_{k}\right)\right)^{2}}\left|f\left(\frac{j_{k}}{\tau}\right)\right|^{2}
$$

When $\alpha=n+\frac{1}{2}, p=-n-1$, where $n$ is a negative integer, Theorem 1 and Theorem 2 respectively give in view of [1, Formula (19)], the following corollaries. 
Corollary 1. Let $n$ be a negative integer, $f$ a function such that $f(z)+f(-z)$ is entire of exponential type $\sigma<2 \tau$ and integrable in the sense of Cauchy on $(0, \infty)$. Then

$$
\int_{\rightarrow 0}^{\rightarrow \infty}(f(x)+f(-x)) d x=\frac{\pi}{\tau} \sum_{k=1}^{\infty} c_{k}\left(f\left(\frac{j_{k}\left(n+\frac{1}{2}\right)}{\tau}\right)+f\left(-\frac{j_{k}\left(n+\frac{1}{2}\right)}{\tau}\right)\right)
$$

where

$$
c_{k}:=\sum_{r=0}^{-n-1} \frac{(-2 n-r-2) !(-2 n-2 r-2) !}{r !\left[2^{-n-r-1}(-n-r-1) !\right]^{2}} j_{k}^{2 n+2 r+2} .
$$

Corollary 2. Let $n$ be a negative integer, then (9) holds provided that $f$ is a function such that $f(z)+f(-z)$ is entire of exponential type $2 \tau$ belonging to $L^{1}[0, \infty)$.

We also mention the following corollary of Theorem 1.

Corollary 3. Let $\alpha \in \mathbb{C} \backslash\{-1,-2, \ldots\}, f$ a function such that $f(z)-f(-z)$ is entire of exponential type $\sigma<2 \tau$ and $x^{2 \alpha+2 p}(f(x)-f(-x))$ is integrable in the sense of Cauchy on $(0, \infty)$. Then

$$
\begin{aligned}
\int_{\rightarrow 0}^{\rightarrow \infty} & x^{2 \alpha+2 p}(f(x)-f(-x)) d x \\
& =\frac{2}{\tau^{2 \alpha+2 p+1}} \sum_{k=1}^{\infty} \frac{j_{k}^{2 p-1}}{\left(G_{\alpha}^{\prime}\left(j_{k}\right)\right)^{2}}\left(f\left(\frac{j_{k}}{\tau}\right)-f\left(-\frac{j_{k}}{\tau}\right)\right) .
\end{aligned}
$$

This result is obtained by applying Theorem 1 to the function $(f(z)-f(-z)) / z$. Analogous results, where the condition on $f(z)+f(-z)$ is replaced by a condition on $(f(z)-f(-z)) / z$, can be deduced from Theorem 2 and Corollaries 1, 2 .

\section{LEMMAS}

As a special case of $[7$, Lemma 1$]$, we have

Lemma 1. Let $f$ be regular and of exponential type $\sigma$ in the closed upper half-plane. If for some real number $\delta, f(x)=O\left(|x|^{-\delta}\right), x \rightarrow \pm \infty$, then we have

$$
f\left(R e^{i \theta}\right)=O\left(\frac{e^{\sigma R|\sin \theta|}}{R^{\delta}}\right) \text { uniformly for } 0 \leq \theta \leq \pi \text { and } R \rightarrow \infty .
$$

We shall need the following result [8, Lemma 3], for whose proof we refer the reader to $[6, \S 2.2]$.

Lemma 2. Let $f$ be regular and of exponential type in the open right half-plane. If $f$ is integrable in the sense of Cauchy on $[1, \infty)$, then $f(x) \rightarrow 0$ as $x \rightarrow \infty$ and so $f(x)$ is bounded for $x \geq 1$.

Lemma 3. Let $f$ be regular and of exponential type $\sigma$ in the first quadrant. If for some real number $\delta, f(x)=o\left(|x|^{-\delta}\right), x \rightarrow \infty$, then for $\theta$ belonging to any compact subset of $\left[0, \frac{\pi}{2}\right)$, we uniformly have

$$
f\left(\operatorname{Re}^{i \theta}\right)=o\left(\frac{e^{\sigma R|\sin \theta|}}{R^{\delta}}\right) \quad \text { as } \quad R \rightarrow \infty .
$$

Proof. Apply [3, Theorem 6.2.8] to the function $G(z):=(z+i)^{\delta} f(z)$ which is regular and of exponential type $\sigma$ in the first quadrant, and $G(x) \rightarrow 0$ as $x \rightarrow \infty$. 
Let

$$
R_{N}=R_{N}(\alpha):=N \pi+\Re(\alpha) \pi / 2+\pi / 4
$$

We need the following result [5, Lemma 1$]$.

Lemma 4. Let $z=R e^{i \theta}$ be a complex number on the the circle $|z|=R_{N}$, where $N$ is a large positive integer. There exists a positive constant $C=C(\alpha)$ such that

$$
\left|J_{\alpha}(z)\right|>\frac{C}{\sqrt{R_{N}}} e^{R_{N}|\sin \theta|}, \quad|\theta| \leq \pi .
$$

We need to recall that

$$
H_{\alpha}^{(1)}(z):=\frac{J_{-\alpha}(z)-e^{-\alpha \pi i} J_{\alpha}(z)}{i \sin (\alpha \pi)} \text { and } H_{\alpha}^{(2)}(z):=\frac{e^{\alpha \pi i} J_{\alpha}(z)-J_{-\alpha}(z)}{i \sin (\alpha \pi)}
$$

are said to be the Bessel functions of the third kind of order $\alpha$. When $\alpha$ is an integer, the right-hand sides are to be replaced by their limits $[11, \S 3.6]$. In order to prove the next lemma, we shall need the following asymptotic expansions of these functions $[11, \S 7.2]$.

$$
\begin{gathered}
H_{\alpha}^{(1)}(z)=\sqrt{\frac{2}{\pi z}} e^{i\left(z-\frac{\alpha \pi}{2}-\frac{\pi}{4}\right)}\left(1+O\left(|z|^{-1}\right)\right), \quad \Im(z) \geq 0, \quad|z| \rightarrow \infty, \\
H_{\alpha}^{(2)}(z)=\sqrt{\frac{2}{\pi z}} e^{-i\left(z-\frac{\alpha \pi}{2}-\frac{\pi}{4}\right)}\left(1+O\left(|z|^{-1}\right)\right), \quad \Im(z) \leq 0, \quad|z| \rightarrow \infty .
\end{gathered}
$$

We also need to introduce $C_{\lambda}=\{z:|z|=\lambda\}, C_{\lambda}^{+}:=\{z:|z|=\lambda, \Im(z) \geq 0\}$ and $C_{\lambda}^{-}:=\{z:|z|=\lambda, \Im(z) \leq 0\}$. From now on, for all large $R_{N}$, we give to the paths $C_{R_{N}}, C_{R_{N}}^{+}$and $C_{R_{N}}^{-}$the positive orientation, and for all small $\varepsilon>0$, we give to $C_{\varepsilon}, C_{\varepsilon}^{+}$and $C_{\varepsilon}^{-}$, the negative orientation. We are now ready to state the following

Lemma 5. Let $m$ be a positive integer and $\alpha, \beta \in \mathbb{C}$. If $f$ is an even entire function of exponential type $\sigma<2 m$ such that $x^{\Re(\beta)} f(x)$ is integrable in the sense of Cauchy on $(0, \infty)$, then

$$
\lim _{N \rightarrow \infty} \int_{C_{R_{N}}^{+}} z^{\beta} f(z)\left(\frac{H_{\alpha}^{(1)}(z)}{J_{\alpha}(z)}\right)^{m} d z=0
$$

and

$$
\lim _{N \rightarrow \infty} \int_{C_{R_{N}}^{-}} z^{\beta} f(z)\left(\frac{H_{\alpha}^{(2)}(z)}{J_{\alpha}(z)}\right)^{m} d z=0 .
$$

Proof. By virtue of (14) there exist positive constants $C_{1}$ and $R_{1}^{\prime}$ such that

$$
\left|H_{\alpha}^{1}\left(R e^{i \theta}\right)\right| \leq \frac{C_{1}}{\sqrt{R}}\left|e^{i R e^{i \theta}}\right|=\frac{C_{1}}{\sqrt{R}} e^{-R \sin \theta} \quad \text { for all } \quad R \geq R_{1}^{\prime} .
$$

Applying Lemma 2 to the function $F(z):=z^{\Re(\beta)} f(z)$, we conclude using the parity of $f$ that $F(z) \rightarrow 0$ as $x \rightarrow \pm \infty$, which is equivalent to

$$
|f(x)|=o\left(|x|^{-\Re(\beta)}\right) \quad \text { as } \quad x \rightarrow \pm \infty .
$$

Therefore by Lemma 1 applied to $f(z)$ and $F_{1}(z):=\overline{f(\bar{z})}$, there exist two constants $M>0$ and $R_{2}^{\prime}>R_{1}^{\prime}$ such that for all $R \geq R_{2}^{\prime}$ we have

$$
R^{\Re(\beta)}\left|f\left(R e^{i \theta}\right)\right| \leq M e^{\sigma R|\sin \theta|} \quad \text { for all } \quad \theta \in[-\pi, \pi] .
$$


According to (19) and the hypothesis, we may apply Lemma 3 to $f(z)$ and $F_{1}(z)$ to deduce that

$$
f\left(R e^{i \theta}\right)=o\left(\frac{e^{\sigma R|\sin \theta|}}{R^{\Re(\beta)}}\right) \text { as } R \rightarrow \infty,
$$

uniformly in $\theta$ belonging to any compact subset of $[-\pi, \pi] \backslash\left\{\frac{-\pi}{2}, \frac{\pi}{2}\right\}$.

Now let $C$ be as in Lemma 4 and

$$
\varepsilon^{\prime}:=\frac{\varepsilon C^{m}(2 m-\sigma)}{C_{1}^{m} \pi},
$$

where $\varepsilon$ is any given positive number. From (21) it follows that for any given $\delta$ in $\left(0, \frac{\pi}{2}\right)$, there exists $R_{3}^{\prime}>R_{2}^{\prime}$, such that for $R \geq R_{3}^{\prime}$

$$
R^{\Re(\beta)}\left|f\left(R e^{i \theta}\right)\right| \leq \varepsilon^{\prime} e^{\sigma R|\sin \theta|} \quad \text { for all } \quad \theta \in[-\delta, \delta] \cup[\pi-\delta, \pi+\delta] .
$$

Next, suppose $R=R_{N} \geq R_{3}^{\prime}$, then using (18), (20), (22) and Lemma 4, we obtain

$$
\begin{aligned}
I(R) & :=\left|\int_{C_{R}^{+}} z^{\beta} f(z)\left(\frac{H_{\alpha}^{(1)}(z)}{J_{\alpha}(z)}\right)^{m} d z\right| \\
& \leq \int_{0}^{\pi}\left|\left(R e^{i \theta}\right)^{\beta} f\left(R e^{i \theta}\right)\right|\left(\frac{\left|H_{\alpha}^{(1)}\left(R e^{i \theta}\right)\right|}{\left|J_{\alpha}\left(R e^{i \theta}\right)\right|}\right)^{m} R d \theta \\
& \leq\left(\int_{0}^{\delta}+\int_{\pi-\delta}^{\pi}\right) \varepsilon^{\prime} e^{\sigma R|\sin \theta|}\left(\frac{C_{1} e^{-R \sin \theta}}{C e^{R|\sin \theta|}}\right)^{m} R d \theta \\
& =\frac{\varepsilon(2 m-\sigma)}{\pi}\left(\int_{\delta}^{\pi-\delta}+\int_{\pi-\delta}^{\pi}\right) e^{(\sigma-2 m) R|\sin \theta|} R d \theta \\
& +M \frac{C_{1}^{m}}{C^{m}} \int_{\delta}^{\pi-\delta} e^{(\sigma-2 m) R|\sin \theta|} R d \theta \\
& =\frac{2 \varepsilon(2 m-\sigma)}{\pi} \int_{0}^{\delta} e^{(\sigma-2 m) R|\sin \theta|} R d \theta+2 M \frac{C_{1} e^{-R \sin \theta}}{C^{m}} \int_{\delta}^{m} e^{\frac{\pi}{2}} e^{(\sigma-2 m)} R d \theta \sin \theta \mid R d \theta
\end{aligned}
$$

since in view of the periodicity and the parity of $\theta \mapsto|\sin \theta|$, we have

$$
\int_{\pi-\delta}^{\pi} e^{(\sigma-2 m) R|\sin \theta|} d \theta=\int_{-\delta}^{0} e^{(\sigma-2 m) R|\sin \theta|} d \theta=\int_{0}^{\delta} e^{(\sigma-2 m) R|\sin \theta|} d \theta,
$$

and

$$
\int_{\frac{\pi}{2}}^{\pi-\delta} e^{(\sigma-2 m) R|\sin \theta|} d \theta=\int_{-\frac{\pi}{2}}^{-\delta} e^{(\sigma-2 m) R|\sin \theta|} d \theta=\int_{\delta}^{\frac{\pi}{2}} e^{(\sigma-2 m) R|\sin \theta|} d \theta .
$$

Thus, using the inequality $\sin \theta \geq 2 \frac{\theta}{\pi}$ for $\theta \in\left[0, \frac{\pi}{2}\right]$, we obtain

$$
\begin{aligned}
I(R) & \leq \frac{2 \varepsilon(2 m-\sigma)}{\pi} \int_{0}^{\delta} e^{(\sigma-2 m) R \frac{2}{\pi} \theta} R d \theta+2 M \frac{C_{1}^{m}}{C^{m}} \int_{\delta}^{\frac{\pi}{2}} e^{(\sigma-2 m) R \frac{2}{\pi} \theta} R d \theta \\
& =\varepsilon\left(1-e^{(\sigma-2 m) R \frac{2}{\pi} \delta}\right)+\frac{M C_{1}^{m} \pi}{C^{m}(2 m-\sigma)}\left(e^{(\sigma-2 m) R \frac{2}{\pi} \delta}-e^{(\sigma-2 m) R}\right) .
\end{aligned}
$$


On the other hand, since $\sigma<2 m$, we have

$$
0 \leq\left(1-e^{(\sigma-2 m) R \frac{2}{\pi} \delta}\right) \leq 1 \text { and } \lim _{R \rightarrow \infty}\left(e^{(\sigma-2 m) R \frac{2}{\pi} \delta}-e^{(\sigma-2 m) R}\right)=0 .
$$

It follows that for $R=R_{N}$ sufficiently large, we get $I(R) \leq 2 \varepsilon$, which proves (16).

In the same manner, we use (15), (20), (22) and Lemma 4 to prove (17).

Lemma 6. Let $\alpha \in \mathbb{C}$ and let $f$ be an even entire function such that $x^{2 \Re(\alpha)+1} f(x)$ is integrable in the sense of Cauchy on $(0, \infty)$. Then we have

$$
\lim _{\varepsilon \rightarrow 0} \int_{C_{\varepsilon}^{+}} z^{2 \alpha+1} f(z) d z=0
$$

$$
\begin{aligned}
& \text { if } \alpha \text { is an integer, then for } R>0 \\
& \lim _{\beta \rightarrow \alpha} \int_{0}^{R} x^{2 \beta+1} f(x) d x=\int_{0}^{R} x^{2 \alpha+1} f(x) d x .
\end{aligned}
$$

Proof. Let $q$ be the smallest nonnegative integer such that $f^{(q)}(0) \neq 0$ and consider $a_{q}:=f^{q}(0) / q$ ! . Then $x^{2 \Re(\alpha)+1} f(x)$ is equivalent in a neighbourhood of zero to the function $a_{q} x^{q+2 \Re(\alpha)+1}$. Since by assumption $x^{2 \Re(\alpha)+1} f(x)$ is integrable in the sense of Cauchy on $(0,1]$, the same must be true about $a_{q} x^{q+2 \Re(\alpha)+1}$ and so

$$
\mu:=q+2 \Re(\alpha)+2>0 .
$$

Since we may write $f(z):=z^{q} h(z)$, where $h(z)$ is entire, there exists a positive constant $N$, such that

$$
|f(z)| \leq N|z|^{q} \quad \text { if } \quad|z| \leq 1 .
$$

Hence we conclude that for $\varepsilon$ in $(0,1)$

$$
\left|\int_{C_{\varepsilon}^{+}} z^{2 \alpha+1} f(z) d z\right| \leq \int_{C_{\varepsilon}^{+}}|z|^{2 \Re(\alpha)+1}|f(z)||d z| \leq N \pi \varepsilon^{\mu} .
$$

Now letting $\varepsilon$ tend to zero, we readily obtain (i).

When $\alpha$ is integer, (23) implies that $\mu$ is a positive integer, so $\mu \geq 1$. Therefore $z^{2 \alpha+1} f(z)=z^{\mu-1} h(z)$ is entire and consequently bounded on $[0, R]$. Thus (ii) follows by the Lebesgue dominated convergence theorem.

Lemma 7. If $n$ is a nonnegative integer, then we have

$$
\lim _{q \rightarrow \infty} G_{n+\frac{1}{q}}^{\prime}\left(j_{k}\left(n+\frac{1}{q}\right)\right)=G_{n}^{\prime}\left(j_{k}(n)\right) \quad \text { for } \quad k=1,2, \ldots .
$$

Proof. Noting that the positive zeros of $J_{\alpha}(z)$ vary continuously as $\alpha$ varies for $\alpha>-1$, we have

$$
\lim _{q \rightarrow \infty} j_{k}\left(n+\frac{1}{q}\right)=j_{k}(n) \quad \text { for } \quad k=1,2, \ldots,
$$

since for $\alpha>-1,\left\{j_{k}(\alpha)\right\}_{k=1}^{\infty}$ is a sequence of positive numbers. Therefore, for any given positive integer $k$, the sequence $\left\{j_{k}\left(n+\frac{1}{q}\right)\right\}_{q=1}^{\infty}$ is bounded. Let

$$
M_{1}=M_{1}(k):=\sup \left\{\left|j_{k}\left(n+\frac{1}{q}\right)\right|: q=1,2, \ldots\right\} .
$$

Next, we recall that the function $G_{\alpha}(z)$ is an analytic function of $z$ as well as of $\alpha$. To get the derivative with respect to $\alpha$, we use its Laurent expansion $\left(\frac{1}{\Gamma(\alpha)}\right.$ is entire). Therefore using the continuity of $G_{\alpha}^{\prime}(z)$ with respect to both $\alpha$ and $z$, 
we deduce that the family of functions $\left\{G_{n+\frac{1}{q}}^{\prime}(z)\right\}_{q=1}^{\infty}$ is uniformly bounded on any compact subset of $\mathbb{C}$ and

$$
\lim _{q \rightarrow \infty} G_{n+\frac{1}{q}}^{\prime}(z)=G_{n}^{\prime}(z) \quad \text { for } \quad z \in \mathbb{C} .
$$

Hence, by using $\left[10\right.$, Theorem 14.6] we conclude that $\left\{G_{n+\frac{1}{q}}^{\prime}(z)\right\}_{q=1}^{\infty}$, converges uniformly on any compact subset of $\mathbb{C}$ to $G_{n}^{\prime}(z)$. Thus

$$
\left|G_{n+\frac{1}{q}}^{\prime}\left(j_{k}\left(n+\frac{1}{q}\right)\right)-G_{n}^{\prime}\left(j_{k}\left(n+\frac{1}{q}\right)\right)\right| \leq \sup _{|z| \leq M_{1}}\left|G_{n+\frac{1}{q}}^{\prime}(z)-G_{n}^{\prime}(z)\right| \rightarrow 0
$$

as $q$ tends to infinity.

By virtue of (25) and the regularity of $G_{\alpha}^{\prime}(z)$ with respect to $\alpha$, we deduce that

$$
\lim _{q \rightarrow \infty} G_{n}^{\prime}\left(j_{k}\left(n+\frac{1}{q}\right)\right)=G_{n}^{\prime}\left(j_{k}(n)\right) .
$$

Since

$$
\begin{aligned}
& \left|G_{n+\frac{1}{q}}^{\prime}\left(j_{k}\left(n+\frac{1}{q}\right)\right)-G_{n}^{\prime}\left(j_{k}(n)\right)\right| \\
& \quad \leq\left|G_{n+\frac{1}{q}}^{\prime}\left(j_{k}\left(n+\frac{1}{q}\right)\right)-G_{n}^{\prime}\left(j_{k}\left(n+\frac{1}{q}\right)\right)\right|+\left|G_{n}^{\prime}\left(j_{k}\left(n+\frac{1}{q}\right)\right)-G_{n}^{\prime}\left(j_{k}(n)\right)\right|,
\end{aligned}
$$

then (24) follows using (26) and (27).

Observing that the proofs of Theorems 1 and 2 in [2] are similar, we may conclude that the remark made by the author in that paper concerning his first theorem, just after its proof, applies also to the second and for the same reasons. Taking into account the notes given in [7, Section 2] about [2, Theorem 2], we are ready to state

Lemma 8. Let $\left\{\lambda_{k}\right\}_{k=1}^{\infty}$ be a sequence of complex numbers, $\delta$ and $\varepsilon$ two positive constants such that $\lambda_{k}=\lambda_{k}^{\prime}+i \lambda_{k}^{\prime \prime}, \lambda_{k}^{\prime}>0, \lambda_{k+1}^{\prime}-\lambda_{k}^{\prime} \geq \delta$ and $\left|\lambda_{k}^{\prime \prime}\right| \leq \varepsilon$. If $f$ is regular and of exponential type in the open right half-plane such that

$$
\int_{0}^{\infty}|f(x)| d x<\infty
$$

then

$$
\sum_{k=1}^{\infty}\left|f\left(\lambda_{k}\right)\right|<\infty
$$

Lemma 9. If $f$ satisfies the conditions of Theorem 2, then the series on the righthand side of (5) is absolutely convergent.

Proof. First we suppose $f$ is even, $\tau=1$ and $p=0$. Since for $k$ sufficiently large [11, p. 506]

$$
j_{k}=\left(k+\frac{\alpha}{2}-\frac{1}{4}\right) \pi-\frac{4 \alpha^{2}-1}{8 \pi\left(k+\frac{\alpha}{2}-\frac{1}{4}\right)}-\frac{\left(4 \alpha^{2}-1\right)\left(28 \alpha^{2}-31\right)}{384 \pi^{3}\left(k+\frac{\alpha}{2}-\frac{1}{4}\right)^{3}}+\ldots,
$$

then we have

$$
\begin{aligned}
& \lambda_{k}^{\prime}:=\Re\left(j_{k}\right)=\left(k+\frac{\Re(\alpha)}{2}-\frac{1}{4}\right) \pi+O\left(\frac{1}{k}\right), \\
& \lambda_{k}^{\prime \prime}:=\Im\left(j_{k}\right)=\Im(\alpha) \frac{\pi}{2}+O\left(\frac{1}{k}\right) .
\end{aligned}
$$


Thus, there exist two positive constants $\delta$ and $\varepsilon$, such that $\lambda_{k+1}^{\prime}-\lambda_{k}^{\prime} \geq \delta$ and $\left|\lambda_{k}^{\prime \prime}\right| \leq \varepsilon$. Therefore applying Lemma 8 to the function $F(z):=z^{2 \alpha+1} f(z)$ with $\lambda_{k}:=j_{k}$, we obtain

$$
\sum_{k=1}^{\infty}\left|F\left(\lambda_{k}\right)\right|=\sum_{k=1}^{\infty}\left|j_{k}\right|^{2 \Re(\alpha)+1}\left|f\left(j_{k}\right)\right|<\infty .
$$

Let $A:=\sup \left\{\left|\Im\left(j_{k}\right)\right|: k=1,2, \ldots\right\}$, then $A$ is finite by (30). Now we recall the recurrence formula $[11, \S 3.2]$

$$
J_{\alpha}^{\prime}(z)=\frac{-\alpha}{z} J_{\alpha}(z)+J_{\alpha-1}(z)
$$

and the asymptotic formula $[11, \S 7.21]$

$$
J_{\alpha}(z)=\sqrt{\frac{2}{\pi z}} \cos \left(z-\frac{\alpha \pi}{2}-\frac{\pi}{4}\right)+O\left(\frac{1}{|z|^{\frac{3}{2}}}\right), \quad|\arg (z)|<\pi, \quad|z| \rightarrow \infty .
$$

Replacing in (32) $J_{\alpha}(z)$ and $J_{\alpha-1}(z)$ by their respective asymptotic expansions, we obtain for $z \in\{z:|\arg (z)|<\pi,|\Im(z)| \leq A\}$

$$
J_{\alpha}^{\prime}(z)=-\sqrt{\frac{2}{\pi z}} \sin \left(z-\frac{\alpha \pi}{2}-\frac{\pi}{4}\right)+O\left(\frac{1}{|z|^{\frac{3}{2}}}\right), \quad|z| \rightarrow \infty,
$$

since $\sup \left\{\cos \left(z-\frac{\alpha \pi}{2}-\frac{\pi}{4}\right):|\Im(z)| \leq A\right\}$ is finite.

It follows from $(28)$ that for all large $k$

$$
\left|\sin \left(j_{k}-\frac{\alpha \pi}{2}-\frac{\pi}{4}\right)\right|>\frac{1}{\sqrt{2}} .
$$

Therefore using (33), we deduce that there exists a constant $K_{1}>0$ such that

$$
\left|J_{\alpha}^{\prime}\left(j_{k}\right)\right| \geq \frac{1}{\sqrt{2 \pi\left|j_{k}\right|}} \quad \text { for all } \quad k \geq K_{1},
$$

and using (6) we have

$$
\sum_{k=1}^{\infty}\left|\frac{f\left(j_{k}\right)}{\left(G_{\alpha}^{\prime}\left(j_{k}\right)\right)^{2}}\right|=\sum_{k=1}^{\infty}\left|\frac{j_{k}^{2 \alpha}}{\left(J_{\alpha}^{\prime}\left(j_{k}\right)\right)^{2}} f\left(j_{k}\right)\right| \leq 2 \pi \sum_{k=1}^{\infty}\left|j_{k}\right|^{2 \Re(\alpha)+1}\left|f\left(j_{k}\right)\right|<\infty .
$$

For the general case when $f(z), \tau$ and $p$ satisfy the conditions of Theorem 2 without any restriction, we may apply the above consideration to the function

$$
g(z):=z^{2 p}\left(f\left(\frac{z}{\tau}\right)+f\left(\frac{-z}{\tau}\right)\right) .
$$

\section{Proofs OF THE THEOREMS}

Proof of Theorem 1. Without loss of generality, we may suppose (as in the proof of Lemma 9) that $f$ is even, $\tau=1$ and $p=0$.

Assume that $\alpha$ is not an integer. We consider the functions

$$
F_{\alpha}(z):=z^{2 \alpha+1} f(z), \quad K(z):=e^{\alpha \pi i} \frac{J_{-\alpha}(z)}{J_{\alpha}(z)} .
$$

Since $G_{\beta}(z)$ is entire for all $\beta$, the function

$$
F_{\alpha}(z) K(z)=e^{\alpha \pi i} z f(z) \frac{G_{-\alpha}(z)}{G_{\alpha}(z)}
$$


is meromorphic in $\mathbb{C}$. Besides, using (28) we have $\left|j_{N}\right|=\left|N \pi+\alpha \frac{\pi}{2}-\frac{\pi}{4}\right|+O\left(\frac{1}{N}\right)$, so after a few calculations we obtain

$$
\begin{gathered}
R_{N}^{2}-\left|j_{N}\right|^{2}=\pi^{2}\left(N+\frac{\Re(\alpha)}{2}\right)-\left(\frac{\Im(\alpha) \pi}{2}\right)^{2}+O(1), \\
\left|j_{N+1}\right|^{2}-R_{N}^{2}=\pi^{2}\left(N+\frac{\Re(\alpha)}{2}\right)+\frac{\pi^{2}}{2}+\left(\frac{\Im(\alpha) \pi}{2}\right)^{2}+O(1) .
\end{gathered}
$$

Hence, there exists $N_{0}>0$, such that

$$
\left|j_{N}\right|<R_{N}<\left|j_{N+1}\right| \quad \text { for } \quad N>N_{0} .
$$

Thus, using the residue theorem, we get for $N>N_{0}$

$$
\begin{aligned}
\int_{C_{R_{N}}} F_{\alpha}(z) K(z) d z & =2 \pi i \sum_{\left|j_{k}\right|<R_{N}} \operatorname{Res}\left(F_{\alpha}(z) K(z)\right)_{z=j_{k}} \\
& =2 \pi i \sum_{\substack{k=-N \\
k \neq 0}}^{N} e^{\alpha \pi i} j_{k} \frac{G_{-\alpha}\left(j_{k}\right)}{G_{\alpha}^{\prime}\left(j_{k}\right)} f\left(j_{k}\right) \\
& =4 \pi i \sum_{k=1}^{N} e^{\alpha \pi i} j_{k} \frac{G_{-\alpha}\left(j_{k}\right)}{G_{\alpha}^{\prime}\left(j_{k}\right)} f\left(j_{k}\right) .
\end{aligned}
$$

But since $[11, \S 3.12]$

$$
J_{\alpha}^{\prime}(z) J_{-\alpha}(z)-J_{\alpha}(z) J_{-\alpha}^{\prime}(z)=\frac{2 \sin (\alpha \pi)}{\pi z},
$$

we have in view of (6) for $N>N_{0}$

$$
\int_{C_{R_{N}}} F_{\alpha}(z) K(z) d z=4\left(e^{2 \alpha \pi i}-1\right) \sum_{k=1}^{N} \frac{f\left(j_{k}\right)}{\left(G_{\alpha}^{\prime}\left(j_{k}\right)\right)^{2}} .
$$

Next, we note using the definitions of $H_{\alpha}^{(1)}(z)$ and $H_{\alpha}^{(2)}(z)$ that

$$
\begin{aligned}
K(z) & =1+\frac{e^{2 \alpha \pi i}-1}{2} \frac{H_{\alpha}^{(1)}(z)}{J_{\alpha}(z)} \\
& =e^{2 \alpha \pi i}-\frac{e^{2 \alpha \pi i}-1}{2} \frac{H_{\alpha}^{(2)}(z)}{J_{\alpha}(z)} .
\end{aligned}
$$

We split the integral over $C_{R_{N}}$ into two integrals over $C_{R_{N}}^{+}$and $C_{R_{N}}^{-}$respectively. On replacing $K(z)$ by the right-hand side of (37) in the first one, and by the righthand side of (38) in the second, (36) gives for $N>N_{0}$

$$
\begin{aligned}
& \int_{C_{R_{N}}^{+}} F_{\alpha}(z) d z+\frac{e^{2 \alpha \pi i}-1}{2} \int_{C_{R_{N}}^{+}} \frac{H_{\alpha}^{(1)}(z)}{J_{\alpha}(z)} f(z) d z \\
& \quad+e^{2 \alpha \pi i} \int_{C_{R_{N}}^{-}} F_{\alpha}(z)-d z \frac{e^{2 \alpha \pi i}-1}{2} \int_{C_{R_{N}}^{-}} \frac{H_{\alpha}^{(2)}(z)}{J_{\alpha}(z)} f(z) d z \\
& =4\left(e^{2 \alpha \pi i}-1\right) \sum_{k=1}^{N} \frac{f\left(j_{k}\right)}{\left(G_{\alpha}^{\prime}\left(j_{k}\right)\right)^{2}} .
\end{aligned}
$$

Let us denote by $\Gamma$ the union of the two intervals $\left[-R_{N},-\varepsilon\right],\left[\varepsilon, R_{N}\right]$ and the two semicircles $C_{R_{N}}^{+}, C_{\varepsilon}^{+}$. By choosing the logarithm whose imaginary part belongs to 
the interval $\left(-\frac{\pi}{2}, \frac{3 \pi}{2}\right]$, it is clear that $F_{\alpha}(z)$ is regular in a simply connected open set containing $\Gamma$. Hence, using Cauchy's theorem, we have

$$
\begin{aligned}
\int_{C_{R_{N}}^{+}} F_{\alpha}(z) d z & =-\int_{-R_{N}}^{-\varepsilon} F_{\alpha}(x) d x-\int_{C_{\varepsilon}^{+}} F_{\alpha}(z) d z-\int_{\varepsilon}^{R_{N}} F_{\alpha}(x) d x \\
& =-\int_{\varepsilon}^{R_{N}}\left(F_{\alpha}(x)+F_{\alpha}(-x)\right) d x-\int_{C_{\varepsilon}^{+}} F_{\alpha}(z) d z .
\end{aligned}
$$

But since $f(x)$ is even, we obtain

$$
\int_{C_{R_{N}}^{+}} F_{\alpha}(z) d z=-\left(1-e^{2 \alpha \pi i}\right) \int_{\varepsilon}^{R_{N}} F_{\alpha}(x) d x-\int_{C_{\varepsilon}^{+}} F_{\alpha}(z) d z .
$$

On the other hand,

$$
\begin{aligned}
e^{2 \alpha \pi i} & \int_{C_{R_{N}}^{-}} z^{2 \alpha+1} f(z) d z=\int_{C_{R_{N}}^{-}}-(-z)^{2 \alpha+1} f(z) d z \\
& =-\int_{C_{R_{N}}^{-}}(-z)^{2 \alpha+1} f(-z) d z=-\int_{\pi}^{2 \pi} F_{\alpha}\left(-R_{N} e^{i \theta}\right) i R_{N} e^{i \theta} d \theta \\
& =\int_{0}^{\pi} F_{\alpha}\left(R_{N} e^{i \phi}\right) i R_{N} e^{i \phi} d \phi=\int_{C_{R_{N}}^{+}} F_{\alpha}(z) d z .
\end{aligned}
$$

Hence, letting $\varepsilon$ tend to zero in (40) and using part (i) of Lemma 6 , we deduce that

$$
e^{2 \alpha \pi i} \int_{C_{R_{N}}^{-}} F_{\alpha}(z) d z=\int_{C_{R_{N}}^{+}} F_{\alpha}(z) d z=\left(e^{2 \alpha \pi i}-1\right) \int_{\rightarrow 0}^{R_{N}} F_{\alpha}(x) d x .
$$

Using (39) and (41), we obtain when $\alpha$ is not an integer and $N>N_{0}$

$$
\begin{gathered}
\int_{\rightarrow 0}^{R_{N}} F_{\alpha}(x) d x+\frac{1}{4} \int_{C_{R_{N}}^{+}} \frac{H_{\alpha}^{(1)}(z)}{J_{\alpha}(z)} f(z) d z-\frac{1}{4} \int_{C_{R_{N}}^{-}} \frac{H_{\alpha}^{(2)}(z)}{J_{\alpha}(z)} f(z) d z \\
=2 \sum_{k=1}^{N} \frac{f\left(j_{k}\right)}{\left(G_{\alpha}^{\prime}\left(j_{k}\right)\right)^{2}} .
\end{gathered}
$$

If $n$ is a given nonnegative integer, then by virtue of (35), we have $G_{n}(z) \neq 0$ on $C_{R_{N}}=C_{R_{N}(n)}$. Besides, the function $G_{\alpha}(z)$ is analytic of $\alpha$ as well of $z$. Therefore $1 / J_{\alpha}(z)$ is bounded in a neighbourhood of the set $\left\{(\alpha, z): \alpha=n, z \in C_{R_{N}(n)}\right\}$. Using this and formulae (14), (15) we obtain for $q$ sufficiently large

$$
\begin{aligned}
& \sup \left\{\left|H_{\alpha}^{(1)}(z) / J_{\alpha}(z)\right|: z \in C_{R_{N}(\alpha)}^{+}, n-\frac{1}{q} \leq \alpha \leq n+\frac{1}{q}\right\}<\infty, \\
& \sup \left\{\left|H_{\alpha}^{(2)}(z) / J_{\alpha}(z)\right|: z \in C_{R_{N}(\alpha)}^{-}, n-\frac{1}{q} \leq \alpha \leq n+\frac{1}{q}\right\}<\infty .
\end{aligned}
$$

Further, let $\alpha=\alpha_{q}:=n+\frac{1}{q}$ in formula (42); then by letting $q$ tend to infinity, we formally obtain (42) for $\alpha=n$. To justify the interchanges of the order of integration and limit, we use part (ii) of Lemma 6 for the first integral. For the second and the third we use (43), (44) respectively and the Lebesgue dominated convergence theorem. The interchange of the order of summation and limit on the right-hand side of (42) is justified using Lemma 7 and the continuity of $f(x)$. 
Now letting $N$ tend to infinity in (42) and applying Lemma 5 with $m=1, \beta=$ $2 \alpha+1$, we deduce that

$$
\int_{\rightarrow 0}^{\rightarrow \infty} F_{\alpha}(x) d x=2 \sum_{k=1}^{\infty} \frac{f\left(j_{k}\right)}{\left(G_{\alpha}^{\prime}\left(j_{k}\right)\right)^{2}}
$$

which is equivalent to formula (4) when $f(z)$ is even, $\tau=1$ and $p=0$.

Proof of Theorem 2. Without loss of generality, we may suppose that $f$ is even, $\tau=1$ and $p=0$. Let

$$
F(z):=z^{2 \alpha+1} f(z) \quad, \quad \psi_{\tau}\left(j_{k}\right):=\frac{2}{\tau^{2 \alpha+2}\left(G_{\alpha}^{\prime}\left(j_{k}\right)\right)^{2}} f\left(\frac{j_{k}}{\tau}\right) \quad \text { for } \quad k= \pm 1 \pm 2, \ldots .
$$

Then according to hypothesis, the function $f(z)$ satisfies the conditions of Theorem 1 with $p=0$ and $\tau=1+\varepsilon$, where $\varepsilon$ is a given positive number. We therefore have

$$
\int_{0}^{\infty} F(x) d x=\sum_{k=1}^{\infty} \psi_{1+\varepsilon}\left(j_{k}\right) .
$$

Further, we show that for $\delta>0$ we can choose $K_{0}$ such that

$$
S_{\varepsilon}:=\sum_{k=K_{0}}^{\infty}\left|\psi_{1+\varepsilon}\left(j_{k}\right)\right|<\frac{\delta}{3}
$$

if $\varepsilon \in\left[0, \frac{1}{2}\right]$. By virtue of (29) and (30) there exists $K_{2}$ such that for $\varepsilon \in\left[0, \frac{1}{2}\right]$,

$$
\begin{aligned}
\frac{1}{1+\varepsilon}\left(\Re\left(j_{k+1}\right)-\Re\left(j_{k}\right)\right) & >\frac{1}{2} \\
\left|\frac{\Im\left(j_{k}\right)}{(1+\varepsilon)}-\frac{\Im(\alpha) \pi}{2}\right| & <\frac{1}{2} \text { for } k \geq K_{2} .
\end{aligned}
$$

Let

$$
I_{k}:=\left[k-\frac{1}{2}, k+\frac{1}{2}\right], I:=\left[\frac{\Im(\alpha) \pi}{2}-\frac{1}{2}, \frac{\Im(\alpha) \pi}{2}+\frac{1}{2}\right],
$$

and let $\xi_{k}(1 \leq k<\infty)$ be a number in $I_{k} \times I$, such that

$$
\left|F\left(\xi_{k}\right)\right|=\max _{z \in I_{k} \times I}|F(z)| .
$$

The points $\xi_{k} \in\left[k-\frac{1}{2}, k\right) \times I$ form a subsequence $\left\{\xi_{k}^{\prime}\right\}$ and those in $\left[k, k+\frac{1}{2}\right] \times I$ form another subsequence $\left\{\xi_{k}^{\prime \prime}\right\}$. Note that $\Re\left(\xi_{n+1}^{\prime}\right)-\Re\left(\xi_{n}^{\prime}\right) \geq \frac{1}{2},\left|\Im\left(\xi_{n}^{\prime}\right)-\frac{\Im(\alpha) \pi}{2}\right| \leq \frac{1}{2}$, and that the same inequalities are also valid if we replace $\xi_{n}^{\prime}$ by $\xi_{n}^{\prime \prime}$. So by Lemma 8 , we have

$$
\sum_{n=1}^{\infty}\left|F\left(\xi_{n}^{\prime}\right)\right|<\infty \quad, \quad \sum_{n=1}^{\infty}\left|F\left(\xi_{n}^{\prime \prime}\right)\right|<\infty .
$$

Thus

$$
\sum_{k=1}^{\infty}\left|F\left(\xi_{k}\right)\right|<\infty .
$$

On the other hand, using Lemma 9, we have

$$
\sum_{k=1}^{\infty}\left|\psi_{1}\left(j_{k}\right)\right|<\infty \text {. }
$$


Therefore, if $K_{1}$ is the constant used in (34), there exists $K_{0}>\max \left\{K_{1}, K_{2}\right\}$ such that

$$
\sum_{k=\left[\frac{K_{0}}{2}\right]}^{\infty}\left|F\left(\xi_{k}\right)\right|<\frac{\delta}{24 \pi}, \quad \sum_{k=K_{0}}^{\infty}\left|\psi_{1}\left(j_{k}\right)\right|<\frac{\delta}{3} .
$$

Hence, by (6) and (34) we have

$$
S_{\varepsilon} \leq \frac{4 \pi}{1+\varepsilon} \sum_{k=K_{0}}^{\infty}\left|\left(\frac{j_{k}}{1+\varepsilon}\right)^{2 \alpha+1} f\left(\frac{j_{k}}{1+\varepsilon}\right)\right|=\frac{4 \pi}{1+\varepsilon} \sum_{k=K_{0}}^{\infty}\left|F\left(\frac{j_{k}}{1+\varepsilon}\right)\right| .
$$

Since by (48), the rectangles of the form $I_{k} \times I$ contain at most two points of the sequence $\left\{\frac{j_{k}}{1+\varepsilon}\right\}_{k \geq K_{2}}$, we have

$$
\frac{4 \pi}{1+\varepsilon} \sum_{k=K_{0}}^{\infty}\left|F\left(\frac{j_{k}}{1+\varepsilon}\right)\right| \leq 8 \pi \sum_{k=\left[\frac{K_{0}}{2}\right]}^{\infty}\left|F\left(\xi_{k}\right)\right|<\frac{\delta}{3},
$$

which proves (47). It is clear that as $\varepsilon \rightarrow 0$ the function $\frac{1}{(1+\varepsilon)^{2 \alpha+2}} f\left(\frac{z}{1+\varepsilon}\right)$ converges uniformly on all compact subsets of $\mathbb{C}$ to $f(z)$. Therefore, there exists a positive $\varepsilon_{0}$ such that for $\varepsilon \in\left[0, \varepsilon_{0}\right]$

$$
\left|\sum_{k=1}^{K_{0}-1} \psi_{1+\varepsilon}\left(j_{k}\right)-\sum_{k=1}^{K_{0}-1} \psi_{1}\left(j_{k}\right)\right|<\frac{\delta}{3} .
$$

Thus for $\varepsilon \in\left[0, \varepsilon_{0}\right]$, we have by virtue of (47) and the inequality on the right-hand side of $(50)$

$$
\left|\sum_{k=1}^{\infty} \psi_{1+\varepsilon}\left(j_{k}\right)-\sum_{k=1}^{\infty} \psi_{1}\left(j_{k}\right)\right| \leq \frac{\delta}{3}+\sum_{k=K_{0}}^{\infty}\left|\psi_{1+\varepsilon}\left(j_{k}\right)\right|+\sum_{k=K_{0}}^{\infty}\left|\psi_{1}\left(j_{k}\right)\right|<\delta .
$$

Hence,

$$
\lim _{\varepsilon \rightarrow 0} \sum_{k=1}^{\infty} \psi_{1+\varepsilon}\left(j_{k}\right)=\sum_{k=1}^{\infty} \psi_{1}\left(j_{k}\right) .
$$

Therefore, (5) follows by letting $\varepsilon$ tend to zero in (46) .

The absolute convergence of the series on the right-hand side of (5) has already been proved in Lemma 9.

Proof of Theorem 3. Write $f(x)=f_{1}(x)+i f_{2}(x)$, where $f_{1}(x)=\Re(f(x))$ and $f_{2}(x)=\Im(f(x))$ when $x \in \mathbb{R}$. The function $f_{1}^{2}(z)+f_{2}^{2}(z)$ satisfies the conditions of Theorem 2. On the other hand, we have $|f(x)|^{2}=f_{1}^{2}(x)+f_{2}^{2}(x)$. Therefore we have

$$
\begin{aligned}
\int_{0}^{\infty} & x^{2 \alpha+2 p+1}\left(|f(x)|^{2}+|f(-x)|^{2}\right) d x \\
& =\frac{2}{\tau^{2 \alpha+2 p+2}} \sum_{k=1}^{\infty} \frac{j_{k}^{2 p}}{\left(G_{\alpha}^{\prime}\left(j_{k}\right)\right)^{2}}\left(\left|f\left(\frac{j_{k}}{\tau}\right)\right|^{2}+\left|f\left(-\frac{j_{k}}{\tau}\right)\right|^{2}\right),
\end{aligned}
$$

which is equivalent to (8).

Remark 1 . The above proof differs only in some details from that of the corollary of $[1]$. 
Remark 2. The numbers $\alpha=-n \in\{-1,-2, \ldots\}$ are the only complex numbers for which we have $G_{\alpha}(0)=0$ and are also the only values for which $G_{\alpha}$ has a zero of multiplicity greater than one. In fact,

$$
G_{-n}(z)=\sum_{k=n}^{\infty}(-1)^{k} \frac{z^{2 k}}{2^{-n+2 k} k !(k-n) !},
$$

so that the multiplicity of zero is $2 n$. Therefore, at the place in the proof of Theorem 1 , where the residue theorem is applied to the integral $\int_{C_{R_{N}}} F_{\alpha}(z) K(z) d z$, there would be a nonzero contribution coming from the $2 n$-pole at the origin. This will mean a change in the form of the formulae (4) and (5).

\section{REFERENCES}

1. R. Ben Ghanem and C. Frappier, Spherical Bessel functions and explicit quadrature formula, Math. Comp. 66 (1997), 289-296. MR 97c:33005

2. R. P. Boas, Inequalities between series and integrals involving entire functions, J. Indian Math. Soc. 16 (1952), 127-135. MR 14:631e

3. __ Entire functions, Academic Press, New York, 1954. MR 16:914f

4. _ Summation formulas and band-limited signals, Tôhoku Math. J. 24 (1972), 121-125. MR 48:9252

5. C. Frappier and P. Olivier, A quadrature formula involving zeros of Bessel functions, Math. Comp. 60 (1993), 303-316. MR 93d:41025

6. C. Frappier and Q. I. Rahman, Une formule de quadrature pour les fonctions entières de type exponentiel, Ann. Sci. Math. Québec 10 (1986), 17-26. MR 88a:65031

7. R. Gervais, Q. I. Rahman and G. Schmeisser, Representation and approximation of functions via (0, 2)-interpolation, J. Approx. Theory 50 (1987), 89-110. MR 88e:41009

8. G. R. Grozev and Q. I. Rahman, A quadrature formula with zeros of Bessel functions as nodes, Math. Comp. 64 (1995), 715-725. MR 95f:65054

9. Q. I. Rahman and G. Schmeisser, Quadrature formulae and functions of exponential type, Math. Comp. 54 (1990), 245-270. MR 90g:65028

10. W. Rudin, Real and complex analysis, 2nd ed., McGraw-Hill, New York, 1974. MR 49:8783

11. G. N. Watson, A treatise on the theory of Bessel functions, 2nd ed., Cambridge Univ. Press, Cambridge, 1966.

Département de Mathématiques et de Statistique, Université de Montréal, C. P. 6128, Succ. Centre-Ville, Montréal, Québec, Canada H3C 3J7

E-mail address: benghanr@ere.umontreal.ca 